\title{
Extrapulmonary Mycobacterium avium complex infections in immunocompetent patients
}

\author{
Vivian Yim BS, David Sotello MD, Kenneth Nugent MD
}

\begin{abstract}
The Mycobacterium avium complex (MAC) includes ubiquitous bacteria that typically cause infection in immunocompromised patients. This paper reviews the presentation, diagnosis, pathogenesis, and treatment of extrapulmonary MAC infections in immunocompetent patients by compiling information from case reports identified by a PubMed search. Mycobacterium avium complex infections in immunocompetent patients can present primarily with extrapulmonary symptoms, and this makes the diagnosis of MAC infection in these patients more difficult. The American Thoracic Society has not established criteria for the diagnosis of extrapulmonary MAC infections; testing for MAC should be based on clinical suspicion or after the exclusion of all other causative agents. Methods of testing should include molecular and biochemical tests, since these tests provide more definitive identification than routine cultures. Extrapulmonary presentations usually respond well to macrolide based multi-drug regimens started as soon as a MAC infection is identified.
\end{abstract}

Keywords: Mycobacterium avium, infection, diagnosis, treatment

\section{INTRODUCTION}

The Mycobacterium avium complex (MAC) includes several species of nontuberculous mycobacteria (NTM) with $M$. intracellular and $M$. avium being the original members; $M$. chimaera, M. paratuberculosis, and $M$. paraintracellulare, etc., were later identified and added to the complex. ${ }^{1}$ These bacteria are Gram-negative, acid-fast bacilli that commonly cause pulmonary disease in the immunocompromised or HIV-positive individuals. The prevalence of nontuberculous mycobacterial infections has increased from 8.7 to 13.9 cases per 100,000 persons between 2008 and 2013 in five states ${ }^{2}$ and at a yearly rate of $5-8 \%$ in the United States and Canada. ${ }^{3}$ Whether this increase is due to a rising incidence is unknown,

Corresponding author: Vivian Yim

Contact Information: Vivian.h.yim@ttuhsc.edu

DOI: 10.12746/swrccc.v8i35.719 and the study was not limited to immunocompetent patients. Both $M$. avium and $M$. abscessus are clinically relevant species associated with NTM cases. ${ }^{2}$ Hill recently reviewed the methods and difficulties associated with obtaining accurate information about the incidence and prevalence of non-tuberculous mycobacterial infections. ${ }^{4}$ The prevalence of immunocompetent patients developing MAC infections and presenting with extrapulmonary symptoms is increasing and is the focus of this review.

\section{Methods}

The articles used in this review were identified using PubMed as the primary search engine with the MESH database defining each keyword and narrowing the search. "Immunocompetent patients" and "Mycobacterium avium complex" were used to exclude immunocompromised cases and to specify the relevant bacteria; the search was restricted to adults and included articles from August 1, 1990, to August 1, 2019. 
Immunocompetency was further determined case-bycase based on reported history in case reports regarding prior health conditions and risk factors, thereby excluding patients with HIVIAIDS and/or chronic immunosuppressive states. After a thorough review and analysis of the case reports from the initial search, immunocompetent patients with primary NTM pulmonary infections, such as Hot Tub lungs and Lady Windermere syndrome, were excluded due to their well-defined populations, mechanism of acquisition, disease progression, and treatment regimen..$^{5-7}$ Therefore, this review is focused primarily on extra-pulmonary presentations of MAC infections. Characteristics of MAC were obtained through reviews specifically on Mycobacterium aviumintracellulare found through PubMed and included for relevant background information to understand the transmission and presentation. Information on possible diagnostic methods recommended in this review was collected from the methods used in the various case reports summarized in Table 1.

\section{Presentation}

Immunocompetent patients with primary extrapulmonary presentations usually do not have any preexisting lung conditions and present with atypical, milder, and longer disease courses that lead to a complex differential diagnosis. Most cases of NTM infections noted in the literature have pulmonary presentations, but cases presented below show little to no pulmonary symptoms prior to presentation with predominant extrapulmonary course of disease.

Cutaneous Mycobacterium avium infections often have no symptoms prior to the appearance of papule(s) or plaque(s). ${ }^{12,13}$ Cutaneous Mycobacterium intracellulare presents as erythematous patches with non-draining papules and/or nodules that can present on the face, neck, back, and/or extremies..$^{11,12,14}$ In two cases, the infection ran an indolent course, as papules initially appeared about 10-18 years prior with periods of acute recurrence, and the patients sought medical help only when the papules were spreading from the initial region and growing larger in size. ${ }^{12,13}$ Another case of cutaneous MAC led to the formation of edematous plaque on the left thumb with scales and small erosions. ${ }^{15} \mathrm{~A}$ skin biopsy specimen showed an epidermal hyperkeratosis with an inflammatory infiltrate and granuloma formation. If untreated, the lesions have been shown to persist for weeks to years, but the bacteria do not usually disseminate, and, if treated with the correct antibiotics, symptoms will resolve after several months. ${ }^{11,12,14-16}$ According to one dermatology paper, MAC was suspected to be auto-inoculated into an open wound as the patient was a sheep-breeder, and the case was limited to cutaneous spread. ${ }^{13}$

One case reported erythematous swelling with fragmentation of the fourth PIP joint caused by MAC diagnosed as an atypical case of destructive monoarthritis, and another case of septic arthritis was found in the left sternoclavicular joint. ${ }^{14,17}$ Biopsy revealed granulomas, dense fibrosis and occasional foreign giant cells. ${ }^{14}$ Mycobacterium avium complex was determined to be the etiology after MAC was found grew out in cultures from synovial tissue, and the patient had negative rheumatoid factor, antinuclear antibodies, and HIV, and had a normal c-reactive protein level and erythrocyte sedimentation rate. ${ }^{14}$ The patient did not respond to NSAID, intramuscular corticosteroid injection, and antibiotics. ${ }^{14}$ Final treatment included isoniazid, pyridoxine, rifabutin, and azithromycin with a good clinical response. ${ }^{14}$

A rare case of mycobacterial spindle cell pseudotumor was reported in the nasal cavity after presenting with nasal obstruction and bloody discharge without a history of long-standing sinus or nasal complications. ${ }^{16}$ Biopsy revealed lymphocytic infiltration in the subepithelial cellular lesion, ${ }^{14}$ and a soft hemorrhagic mass was resected intranasally from the septum, without further note of recurrence or dissemination. ${ }^{16}$ Sotello et al reported that $20 \%$ of NTM infections of the upper extremity $(n=44)$ were secondary to MAC ( $78 \%$ were immunocompetent); the most common associated injury or trauma was local steroid injection and previous surgery. ${ }^{7}$

Infections of MAC that commonly affect immunocompromised patients can also affect immunocompetent patients. Three cases presented with "disseminated" MAC infections, with initial progressive non-specific symptoms of chest pain, chest wall and jaw swelling, or "flu-like" prodrome 1-2 months before presenting to a physician's office with disseminated symptoms. ${ }^{18-20}$ 
Table 1. Studies on Immunocompetent Patients with MAC infections

\begin{tabular}{|c|c|c|c|c|}
\hline & $\begin{array}{l}\text { Demographics/ } \\
\text { Diagnosis }\end{array}$ & Histology & $\begin{array}{l}\text { Chest } \\
\text { Radiography }\end{array}$ & Computed Tomography \\
\hline Case $1^{11}$ & $\begin{array}{l}49 \text { y/o male } \\
\text { Dx: Cutaneous }\end{array}$ & $\begin{array}{l}\text { Epidermal hyperplasia, hyperkeratosis } \\
\text { and perivascular and perifollicular } \\
\text { inflammatory cell infiltrate seen } \\
\text { containing lymph nodes. }\end{array}$ & UR & UR \\
\hline Case $2^{12}$ & $\begin{array}{l}42 \text { y/o male } \\
\text { Dx: Cutaneous }\end{array}$ & $\begin{array}{l}\text { Normal epidermis with nodular } \\
\& \text { non-caseating granulomas } \\
\text { infiltrated by lymphocytes, } \\
\text { histiocytes multinucleate giant cells } \\
\text { and foamy cells. }\end{array}$ & NR & NR \\
\hline Case $3^{15}$ & $\begin{array}{l}66 \text { y/o female } \\
\text { Dx: Cutaneous }\end{array}$ & $\begin{array}{l}\text { Dermal inflammatory infiltrate with } \\
\text { well-formed granulomas. }\end{array}$ & NR & NR \\
\hline Case $5^{16}$ & $\begin{array}{l}83 \text { y/o male } \\
\text { Dx: Mycobacteria } \\
\text { spindle cell } \\
\text { pseudotumor }\end{array}$ & $\begin{array}{l}\text { Spindle-shaped cells with finely } \\
\text { granular and slightly eosinophilic } \\
\text { cytoplasm, with lymphocytes, } \\
\text { histiocyte-like cell infiltrates. }\end{array}$ & UR & Enhancing mass in right nasal cavity \\
\hline Case $6^{14}$ & $\begin{array}{l}74 \text { y/o male } \\
\text { Dx: Destructive } \\
\text { Monoarthritis }\end{array}$ & $\begin{array}{l}\text { Granulomas in dense fibrous tissue } \\
\text { with occasional foreign body giant } \\
\text { cells. }\end{array}$ & NR & NR \\
\hline Case $9^{20}$ & $\begin{array}{l}37 \text { y/o male } \\
\text { Dx: Disseminated } \\
\text { to chest and jaw } \\
\text { swelling }\end{array}$ & $\begin{array}{l}\text { Necrotizing and non-necrotizing } \\
\text { granuloma. }\end{array}$ & NR & NR \\
\hline Case $10^{27}$ & $\begin{array}{l}63 \text { y/o female } \\
\text { Dx: Disseminated } \\
\text { to bones }\end{array}$ & NR & $\begin{array}{l}\text { Slightly enlarged } \\
\text { cardiac shadow and } \\
\text { infiltration on right } \\
\text { lower lobe of lung } \\
\text { w/pleural effusion }\end{array}$ & $\begin{array}{l}\text { MRI: Pericardial effusion and } \\
\text { bilateral pleural effusion. } \\
\text { Bone Scan: Multiple sites of increased } \\
\text { radioactive uptake on vertebral } \\
\text { bodies, right scapula, bilateral femurs, } \\
\text { ribs and pelvis. }\end{array}$ \\
\hline
\end{tabular}

UR-Unremarkable; Dx-Diagnosis; NR-Not reported; P-Pulmonary; AFB-Acid-fast bacilli 
One case involved patient presenting with tachycardia, dyspnea, and atypical chest pain; the MRI revealed pericardial effusion, and a biopsy revealed perivascular lymphocytic inflammatory infiltrate. ${ }^{19}$ Mycobacterium avium complex was identified using gel electrophoresis analysis, and treatment continued for a year with clarithromycin, ethambutol, and prednisone with complete resolution and no relapses. ${ }^{19}$ Another case presented with acute disseminated encephalomyelitis (ADEM), an immune-mediated inflammatory disorder of the central nervous system involving demyelination of white matter and spinal cord. ${ }^{18}$ This patient presented with myalgias, decreased motor strength, disorientation, ataxic gait, and meningitis. ${ }^{18}$ Mycobacterium avium complex was identified by PCR after extensive lab testing, and the patient was started on anti-tuberculous therapy with rifampin, streptomycin, clarithromycin, and methylprednisolone with good clinical recovery and no relapses. ${ }^{18}$ The third case involved a patient presenting with 2 months of chest wall and jaw swelling with lower back pain of 1 day duration and other symptoms suggestive of tuberculous infection. ${ }^{20}$ Mediastinal lymph node biopsy revealed granulomas, and the diagnosis was made based on left chest wall aspirate culture that grew Mycobacterium avium-intracellulare..$^{20}$ The patient was started on anti-tuberculous treatment but was lost to follow up after 2 months. ${ }^{20}$

Reports of extrapulmonary diseases from MAC infection of immunocompetent patients are varied in terms of their presentation, but most cutaneous infections are typically mild and easily treated with a macrolide regimen. Disseminated MAC can require a longer treatment with an anti-tuberculous regimen. There are not enough cases discussed within the scope of this review to come to conclusions using statistical analyses, but there seems to be a predilection for NTM in men.

\section{Diagnosis}

The variety of presentations with atypical MAC infections in immunocompetent patients may delay the diagnosis of MAC infections based on clinical presentation alone. The American Thoracic Society and the Infectious Diseases Society of America have not published a standard protocol for the diagnostic criteria and testing when suspecting an extrapulmonary MAC infection. Many confirmed cases of immunocompetent patients with MAC infections have reported that the initial culture and/or staining processes yielded negative results. ${ }^{15,18,19,21-23}$ Therefore, a differential diagnosis with MAC should not be dismissed simply based on initial staining results, especially if other common disorders are excluded. Negative results may be common due to mycobacterial species special growth condition requirements and a longer incubation period (3-6 weeks). ${ }^{3}$ Therefore, clinicians should consider ordering a second culture or histology and cultures from a biopsy sample to identify possible MAC infections. For example, two cases of primary pulmonary disease caused by Mycobacterium avium were diagnosed using either repeat sputum collection or bronchoscopy with biopsies. ${ }^{23,25}$ Extrapulmonary cases have depended primarily on specimens obtained from synovial, epidermal, or pericardial tissues to isolate Mycobacterium avium. 12,15-20,22,26

Infected tissue commonly shows granulomatous inflammation with lymphocytic infiltration, but no single presentation is specific for MAC infections in immunocompetent patients. ${ }^{13-16,20,21}$ For example, two cases involving the brain and the heart did not show granuloma formation. ${ }^{18,19}$

Rather than relying on culture or histology, a more reliable method of detecting the presence of MAC is through molecular methods using a DNA probe or $16 \mathrm{~S}$ rRNA sequence analysis. In one case, PCR-Restriction Fragment Length Polymorphism identified the subtype Mycobacterium intracellulare by amplifying specific fragments of $h s p 65$ and $16 S$ rDNA and then comparing the sequences using the BLAST program against the pattern of an isolated MAI strain in the GenBank database to reveal $99 \%$ and $100 \%$ homology, respectively. ${ }^{12}$ Polymerase chain reaction followed by a suspension assay identification system that detects the most common 17 species of mycobacteria was also successful in definitively identifying MAC as the cause of infection, ${ }^{15,19}$ more so when the DNA probe failed. ${ }^{24}$ The sensitivity and specificity of PCR-suspension array are $97.1 \%$ and $98.8 \%$, respectively. ${ }^{15}$ Another case used 16S rRNA gene sequencing to identify a MAC isolate of the right posterior lower lobe of the lung. ${ }^{21}$ Molecular methods using biochemical DNA 
probing, PCR, and 16S rRNA sequencing have been effective in confirming MAC infection, and although ATS does not have guidelines related to using molecular methods as diagnostic measures, this approach can be considered based on their reported success.

\section{TREATMENT}

Macrolides have very good activity against MAC. Based on both ATS guidelines for NTM lung disease and reported case studies, macrolides-either clarithromycin and azithromycin-have good in vitro and clinical activity against MAC. ${ }^{25}$ Macrolides combined with ethambutol and rifampicin/rifabutin are the recommended therapy for any patients infected with MAC regardless of presentation. ${ }^{25}$ From previous studies, initial macrolide-based regimens were successful, but patients experiencing relapse often have MAC isolates that are macrolide-resistant due to a point mutation in the 23S rRNA gene. ${ }^{25}$ For macrolide-resistant bacteria, macrolide-regimens must be changed to a multidrug regimen with different mechanisms of action for continued efficacy. Susceptibility testing should be performed whenever bacteria are isolated to determine whether regimen should include a macrolideresistant regimen. Evidence suggests a $96 \%$ rate of seroconversion to negative after12 months of treatment with recommended therapy, ${ }^{25}$ in patients on a multi-drug macrolide treatment regimen with no signs of relapse. ${ }^{12,15,19,26}$ Cases with poor responses to drug therapy or for presentations, such as with bronchogenic cysts, require surgical intervention, followed by months long multi-drug macrolide regimen have had good outcomes. ${ }^{21,24}$ In disseminated MAC infections involving the CNS, co-administration of corticosteroids has been shown to dramatically improve outcomes. ${ }^{18}$

\section{CONCLUSION}

The prevalence of MAC infections is increasing due to simple transmission from accessible environmental niches, leading to infections of immunocompromised patients. Mycobacterium avium complex infections of immunocompetent patients are less common, but more cases have been reported during the last several years. Unlike most presentations of
MAC infections, immunocompetent patients can have extrapulmonary symptoms and disease patterns. Once MAC is confirmed through molecular tests or cultures, clinicians should test for drug-resistance and start the recommended macrolide based regimen. Physicians should be aware of the possibility that MAC are potentially invasive bacteria with a wide range of possible presentations in immunocompetent patients, and MAC infection should be kept in the differential diagnosis, especially when all other diagnoses and infectious agents have been excluded.

Article citation: Yim V, Sotello, D, Nugent K. Extrapulmonary Mycobacterium avium complex infections in immunocompetent patients. The Southwest Respiratory and Critical Care Chronicles 2020;8(35):1-6

From: Department of Internal Medicine, Texas Tech University Health Sciences Center, Lubbock, Texas

Submitted: $2 / 29 / 2020$

Accepted: 6/4/2020

Reviewer: Richard Winn MD

Conflicts of interest: none

This work is licensed under a Creative Commons Attribution-ShareAlike 4.0 International License.

\section{REFERENCES}

1. Akram SM, Attia FN. Mycobacterium Avium Intracellulare. StatPearls Publishing; 2019. http://www.ncbi.nlm.nih.gov/ pubmed/28613762. Accessed June 15, 2019.

2. Donohue MJ, Wymer L. Increasing prevalence rate of nontuberculous Mycobacteria infections in five states, 2008-2013. Ann Am Thorac Soc 2016;13(12):2143-2150.

3. Falkinham JO. Reducing Human Exposure to Mycobacterium avium. Ann Am Thorac Soc 2013;10(4):378-382.

4. Hill AR. The Quest for systematic epidemiology of nontuberculous Mycobacterial lung disease in the United States: Closing in on an elusive goal. Ann Am Thorac Soc 2020; 17(2):169-172.

5. Cappelluti E, Fraire AE, Schaefer OP. A case of "hot tub lung" due to Mycobacterium avium complex in an immunocompetent host. Arch Intern Med 2003;163(7):845-848.

6. Kumfer AM, Edriss H. Lady Windermere Syndrome. Vol 5; 2017. https://pulmonarychronicles.com/index.php/ pulmonarychronicles/article/view/402/884. Accessed June $15,2019$. 
7. Sotello D, Garner HW, Heckman MG, et al. Nontuberculous Mycobacterial infections of the upper extremity: 15-year experience at a tertiary care medical center. J Hand Surg Am 2018;43(4):387.e1-387.e8. doi:10.1016/j.jhsa.2017.10.030

8. Ohkusu K, Bermudez LE, Nash KA, et al. Differential virulence of Mycobacterium avium strains isolated from HIV infected patients with disseminated M. avium complex disease J Infect Dis 2004;190(7):1347-1354.

9. McGarvey JA, Bermudez LE. Phenotypic and genomic analyses of the Mycobacterium avium complex reveal differences in gastrointestinal invasion and genomic composition. Infect Immun 2001;69(12):7242-7249. doi:10.1128/ IAI.69.12.7242-7249.2001

10. Nishiuchi $Y$, Iwamoto $T$, Maruyama F. Infection sources of a common non-tuberculous Mycobacterial pathogen, Mycobacterium avium Complex. Front Med 2017;4:27. doi:10.3389/fmed.2017.00027

11. Landriscina A, Musaev $T$, Amin B, et al. A surprising case of Mycobacterium avium complex skin infection in an immunocompetent patient. J Drugs Dermatol 2014;13(12): 1491-1493.

12. Zhou L, Wang H, Feng S, et al. Cutaneous Mycobacterium intracellulare infection in aniImmunocompetent person. Acta Derm Venereol 2013;93(6):711-714.

13. Satta, GR, Francesca C R, Retanda G, et al. Mycobacterium avium complex: cutaneous infection in an immunocompetent host. Acta Derm Venereol 1999;79(3):249-250.

14. Parperis KM, Ryan LM. An unusual cause of destructive monoarthritis. J Clin Rheumatol 2013;19(4):225. doi:10.1097/RHU.0b013e318293dc05

15. Carlos CA, Tang Y-W, Adler DJ,et al. Mycobacterial infection identified with broad-range PCR amplification and suspension array identification. J Cutan Pathol 2012;39(8): 795-797.

16. Ohara K, Kimura T, Sakamoto K, et al. Nontuberculous mycobacteria-associated spindle cell pseudotumor of the nasal cavity: A case report. Pathol Int 2013;63(5):266-271.

17. Vrettos A, Prasinou M, Ahmad E, et al. Mycobacterium aviumintracellulare septic arthritis of the sternoclavicular joint in an immunocompetent host; a three-year follow-up. Indian J Tuberc 2019;66(3):422-426.

18. Okada H, Yoshioka K. Acute disseminated encephalomyelitis associated with meningitis due to Mycobacterium intracellulare. Intern Med 2010;49(19):2113-2116. http://www.ncbi. nlm.nih.gov/pubmed/20930438. Accessed April 5, 2019.

19. Salemi VMC, Nicodemo AC, Mitteldorf CAT, et al. Mycobacterium avium causing cardiac tamponade in an immunocompetent patient. Eur Heart J 2011;32(16):2074-2074.

20. Eneh KK, Zahir M, Mora ME, et al. Disseminated Mycobacterium avium intracellulare infection in an "Immunocompetent" host. South Med J 2010;103(7):693-696.

21. Lin S-H, Lee L-N, Chang Y-L, et al. Infected bronchogenic cyst due to Mycobacterium avium in an immunocompetent patient. J Infect 2005;51(3):e131-e133.

22. Cappelluti E, Fraire AE, Schaefer OP. A Case of "Hot Tub Lung" Due to Mycobacterium avium complex in an immunocompetent host. Arch Intern Med 2003;163(7):845. doi:10.1001/archinte.163.7.845

23. Khoor A, Leslie KO, Tazelaar HD, et al. Diffuse pulmonary disease caused by nontuberculous mycobacteria in immunocompetent people (Hot Tub Lung). Am J Clin Pathol 2001; 115(5):755-762.

24. Kwon YS, Han J, Jung KH, et al. Mycobacterium avium lung disease combined with a bronchogenic cyst in an immunocompetent young adult. Korean J Intern Med 2013;28(1):94. doi:10.3904/KJIM.2013.28.1.94

25. Griffith DE, Aksamit T, Brown-Elliott BA, et al. American Thoracic Society Documents An Official ATS/IDSA Statement: Diagnosis, Treatment, and Prevention of Nontuberculous Mycobacterial Diseases. Am J Respir Crit Care Med 2007; 175:367-416.

26. Watanabe A, Noguchi M, Nishiwaki K, et al. [Infection with Mycobacterium avium presenting as polypoid lesions in left upper-lobe bronchus of an immunocompetent host]. Nihon Kyobu Shikkan Gakkai Zasshi 1996;34(10):1140-1144.

27. Myojo M, Fujiuchi S, Matsumoto H, et al. Disseminated Mycobacterium avium complex (DMAC) in an immunocompetent adult. Int J Tuberc Lung Dis 2003;7(5):498-501. 